NOTA CIENTÍFICA

\title{
Presencia de Cotylophoron cotylophorum (Trematoda, Taramphisto- midae) en bovinos de Loreto, Perú
}

\section{Report of Cotylophoron cotylophorum (Trematoda, Taramphistomidae) in bovine from Loreto, Perú}

1. Instituto Veterinario de Investigaciones Tropicales y de Altura -IVITA-Iquitos, Facultad de Medicina Veterinaria, Universidad Nacional Mayor de San Marcos. Snchez :nofresp@ hotmail.com

2. Laboratorio de Parasitología Facultad de Medicina Veterinaria y Zootecnia. Universidad Peruana Cayetano Heredia. Lima Perú Email Manuel Tantaleán: mtanta-

3. Laboratorio parasitología, $\mathrm{Fa}$ cultad de Medicina Veterinaria, Universidad Nacional Mayor de San Marcos.

4. Servicio Nacional de Sanidad Agraria SENASA -LORETO-Perú

Presentado: 06/05/2008 Aceptado: 15/07/2008

Publicado online: 28/08/2009

\section{Nofre Sánchez P. ${ }^{1}$, Manuel Tantalean V. ${ }^{2}$, Amanda Chávez ${ }^{3}$ y Alfredo Soto $0 .{ }^{4}$}

\section{Resumen}

La paranfistomosis es una infección ocasionada por trematodes pertenecientes al suborden Paramphistomata, algunas de estas especies son parásitos del rúmen y retículo de rumiantes. Uno de los trematodos más frecuentes en todo el mundo es el Cotylophoron que afecta a rumiantes domésticos y silvestres, especialmente a vacas, ovejas, cabras y búfalos especialmente en regiones tropicales y subtropicales. Se realizó la evaluación parasitaria a 61 ejemplares de ganado bovino aparentemente sanos (42 hembras adultos y 19 menores de un año), manejados al pastoreo, en un fundo ganadero ubicado en la cuenca del río Nanay -lquitos. Las heces fueron obtenidas mediante palpación rectal y procesada mediante el método Dennis modificado. Dos vacas fueron sacrificadas para obtener parásitos adultos. Los resultados indican la presencia de Cotylophoron cotylophorum (Fischoeder, 1901) Stiles \& goldeberger, 1910 en los animales estudiados y es un nuevo registro para el Perú, encontrando porcentajes de infección de 62 y $53 \%$ en adultos y terneros respectivamente. El rango de la carga parasitaria (huevo/gramo de heces) en adultos fue $1-44$ y $1-55$ en animales menores de un año. E estudio revela la existencia del trematodo en un grupo de ganado cruzado manejados al pastoreo, aunque se desconoce los efectos negativos que podría estar causando en la reducida ganadería amazónica.

Palabras claves: rumiantes, trematode, Cotylophoron, ganado bovino.

\section{Abstract}

The paramfistomosis is an infection caused by a trematode belonging to the sub order Paramphistomata, some their species are parasitics of the rumen and reticulo of ruminant. One of the most frequent trematodos in the world is Cotylophoron, it affects to domestic and wild ruminant, especially cows, sheep, goats and buffalos in tropical and subtropical regions. We evaluate the parasitism in 61 specimens of apparently healthy cattle (42 adult females and 19 under one year), fed with wild pastures on a farm located on the Nanay River Basin lquitos. The samples of fresh grounds were obtained by means of rectal palpation and processed by means of the method of Dennis modified. Two cows were sacrificed to collect mature parasites. The results indicate the presence of Cotylophoron cotylophorum (Fischoeder, 1901) Stiles \& goldeberger, 1910, in the studied animals, which is a new record from Peru, being percentages of 62 and $53 \%$ respectively in adults and calves. The range of the parasitic load (egg/grams of grounds) in adults was 1-44 and 1-55 in animals smaller than one year. The study reveals the existence of the trematodo in a group of livestock crusader managed to the shepherding, although one ignores the negative effects that it could be causing in the reduced Amazon cattle raising.

Keywords: ruminants, catlle, trematode, Cotylophoron, parasitic
La paranfistomosis es un parasitismo ocasionado por trematodes perteneciente al suborden Paramphistomata, familia Paramphistomidae (Yumaguti, 1971). Esta familia está constituida por una variedad de géneros y especies difíciles de identificar (Mage et al. 2002) que son causantes de enfermedades en diversos animales de los trópicos y subtrópicos como ovinos, bovinos, caprinos y rumiantes no domésticos. Los bovinos jóvenes de más de un año de edad son los más afectados. En el desarrollo del ciclo biológico del parásito interviene como hospedero intermediario un caracol acuático de las familias Planorbidae o Lymnaeidae (Müller et al. 1992). Esta parasitosis es conocida como "fasciolosis intestinal", "fasciolosis gástrica" y está considerada como una enfermedad grave en bovinos en Estados Unidos, Australia e India. En la india, la Republica de Sudáfrica y Australia, la mortalidad puede llegar hasta el 80 o el $96 \%$ de los animales afectados. Los paranfistomos como Paramphistomum y Cotylophoron producen grandes pérdidas económicas al disminuir la conversión alimenticia, pérdida de peso corporal y baja la producción de leche (Kathoon et al. 2003). El bovino se infecta cuando ingieren las metacercarias durante el consumo del pasto. Este parásito en su forma adulta se ubica en el preestómago y no se consideran patógenas; sin embargo, los estadios inmaduros al migrar en el duodeno y el íleon causan enteritis grave, produciendo necrosis y hemorragia
(Dube et al. 2003, Singh y Lakra 1971). La pérdida de proteína hacia el intestino junto con la pérdida del apetito parece ser la consecuencia fisiopatológica más importante. Histológicamente, produce inflamación catarral y hemorragia generalizada en duodeno y del yeyuno, con destrucción de las glándulas intestinales, degeneración de los nódulos linfáticos asociados y otros órganos durante la migración. Estas lesiones van acompañadas de anemia, hipotroteinemia, edema y emaciación. Kathoon et al. (2003) describen un severo daño hepático e hiperplasia del conducto biliar en búfalos causado por Paramphistomun cervi.

Debido a que la paranfistomosis es una parasitosis poco conocida en el Perú, con escasa información y subestimada importancia, es que en el presente trabajo se da a conocer la presencia de Cotylophoron cotylophorum (Fischoeder, 1901) Stiles \& Goldgerger, 1910, un paranfistómido no registrado en Perú pero presente en un predio ganadero en Iquitos, ciudad que cuenta con los factores epidemiológicos(clima, hospedero intermediario y huésped) para el desarrollo y posible incremento como ha ocurrido con otro tremátode semejante (Mage et al. 2002).

En enero de 2005 se colectaron muestras de heces de 42 vacas cruzadas y 19 terneros menores de un año de edad (7 hembras: 12 machos) del fundo Santo Rosa (Iquitos) con la finalidad de 
identificar la presencia de huevos del tremátode de la familia Paramphistomidae; además, se beneficiaron 2 animales adultos del grupo muestreado con el fin de obtener los parásitos adultos. Las muestras fecales fueron colectadas mediante palpación rectal; la identificación de los huevos se hizo por morfometría y micrometría y para la cuantificación de los mismos se aplicó el método de Dennis modificado (Ueno y Goncalvez 1998). Los trematodos adultos fueron colectados y preservados en formol al $10 \%$ y posteriormente aplanados y coloreados con acetocarmín de Semichon para su identificación, de acuerdo a técnicas convencionales y mediante cortes.

El fundo Santo Rosa se encuentra en el caserío San Pablo de Cuyana, distrito de San Juan Bautista Maynas- Loreto, cuya área geográfica forma parte de la cuenca del río Nanay, localizado en el llano amazónico, área no inundable, que se encuentra a $102 \mathrm{~m}$ de altitud; presenta un relieve irregular con presencia de pequeñas quebradas que desembocan en el río Nanay. La temperatura media y máxima 28 y $34,2^{\circ} \mathrm{C}$. Los valores de humedad relativa mínima y máxima promedio 67 y $97 \%$. La precipitación pluvial total promedio mensual 178 a $284 \mathrm{~mm}$. El sistema de manejo es extensivo, utilizando para la alimentación forraje introducido (Brachiaria sp. y Brisanta sp.) para la alimentación.

Los exámenes efectuados revelan la presencia de Cotylophoron cotylophorum, un trematode Paramphistomidae sin registro en nuestro país, pero conocido en otros paises sudamericanos (Pino y Morales 1982, Racioppi et al. 1994). Esta observación es concordante con la literatura consultada identificando a esta especie como una de las más frecuentes en todo el mundo.

De los grupos muestreados el $47 \%$ de los animales menores de 1 año y el 62\% de los adultos estaban infectados. Estos resultados coinciden con diversos autores quienes advierten que esta enfermedad puede afectar a cualquier individuo de las distintas especies, aunque los bovinos jóvenes de más de un año de edad se encuentran especialmente expuestos. La infección en los animales menores de un año registra menor porcentaje que la de adultos, resultado que probablemente esté dado por el periodo de prepatencia del trematodo y el momento que el animal adquiere la infección. La carga parasitaria (huevo/gramo de heces) registrada es baja, encontrando entre 1 a 44 huevos en adultos y de 1 a 55 en menores de un ańo.
Por ser la paramfistomosis una parasitosis de importancia en la ganadería, se recomienda ampliar los estudios de casos clínicos y de prevalencia para conocer el papel que podría estar jugando en nuestra pequeńa población ganadera de Loreto. Asimismo, la identificación del huésped intermediario es de vital necesidad para establecer un programa de control.

\section{Agradecimientos}

A la familia Vela Reátegui, propietarios del fundo Santa Rosa, por facilitarnos las muestras de estudio y su gran interés de conocer esta parasitosis.

\section{Literatura citada}

Dube S., A. Obiamiwe \& S. Aisen. 2003. Studies on the genus Cotylophoron Fischoeder, 1901 (Paramphistomidae), recovered from nigerian cattle. Folia veterinaria 47: 42-47.

Khatoon N., B. Mujib \& S. Mirza. 2003. Histological changes in the liver of buffaloes by digenetic tremátode Paramphistomun cervi. Pakistan journal of biological sciences 6(17): 1540-1543.

Mage C., H. Bourgne, J-M. Toullieu, D. Rondelaud \& G. Etdreyfuss. 2002. Fasciola hepatica and Paramphistomum daubneyi: changes in prevalences o natural infections in cattle and in Lymnaea truncatula from central France. Vet. res., 33: 439-447.

Müller G. S., I.M. Lara \& P. B Ribeiro. 1992. Infecção natural e experimental de Depanotrema kermatoides (planorbidae) com Paranphistomum sp. (Fischoeder, 1901) no-Rio Grande do Sul, Brasil. Rev. Brasil. Parasitol. Vet. 1:23-26.

Pino L. \& G. Morales. 1982. Lymnaea cubensis Pfeiffer, 1839 hospedero intermediario de Cotylophoron cotylophorum (Fischoeder, 1901) Stiles \& Goldgerger, 1910 en condiciones naturales. Acta Cient. Venezolana 33:57-60.

Racioppi O., O.J. Lombardero \& R.A. Moriena. 1994. Cotylophoron cotylophorum (Fischoeder, 1901) (Trematoda, Paramphistomidae) nuevo parasito para la Argentina, Rev. Med. Vet. (Bs. Aires), 75:228-229.

Singh, D. \& P.Lakra. 1971. Pathologic changes in naturally occurring cotylophoron cotylophorum infection in cattle. Am. J. Vet. Res. 32: 659-663.

Ueno H. \& C. Gonçalves. 1998. Manual para diagnóstico das helmintotes de ruminantes. $4^{\mathrm{a}}$ ed. p 56-57. Japan internacional cooperation agency. 